\title{
Promoción de una alimentación saludable en la infancia y la adolescencia para el cumplimiento de los derechos del niño.
}

DOI: $10.46932 / \mathrm{sfjdv2n2-086}$

Received in: March 1st, 2021

Accepted in: May 30th, 2021

\author{
Raquel María Guevara Ingelmo \\ Doctora en Ciencias Humanas y Sociales
}

Profesora encargada de cátedra e investigadora en Universidad Pontificia de Salamanca Facultad de Educación. C/Henry Collet, 52-70. 37007. Salamanca. España.

E-mail: rmguevarain@upsa.es

\section{José David Urchaga Litago}

Doctor en Psicología

Profesor encargado de cátedra e investigador en Universidad Pontificia de Salamanca

Facultad de Comunicación. C/Henry Collet, 90-98. 37007. Salamanca. España

E-mail: jdurchagali@upsa.es

\section{Fernando González Alonso}

Doctor en Educación

Profesor encargado de cátedra e investigador en Universidad Pontificia de Salamanca

Facultad de Educación. C/Henry Collet, 52-70. 37007. Salamanca. España.

E-mail: fgonzalezal@upsa.es

\section{RESUMEN}

En la convención sobre los derechos del niño se contempla de la necesidad de garantizar el más alto nivel de salud posible en la infancia, siendo además este un objetivo prioritario a nivel educativo y sanitario en el mundo.

La alimentación, es uno de los factores determinantes que más influyen en el estado de salud de las personas, siendo la infancia y la adolescencia periodos clave. La Organización Mundial de la Salud señala que una alimentación inadecuada en la primera infancia es uno de los principales factores de riesgo de mala salud a lo largo de la vida, encontrándose además directamente relacionada con la aparición de enfermedades crónicas en la adultez.

La propia convención citada, señala además como derecho de este grupo de población el acceso a una educación integral que tome en consideración todas las dimensiones de la persona (física, mental y social). Se presenta a continuación un estudio de los hábitos de alimentación de la población adolescente que, fundamentado en estos derechos del niño, justifica la importancia de programas de intervención a nivel educativo que favorezcan hábitos correctos de alimentación desde la infancia como medio de protección y promoción de la salud.

\section{INTRODUCCIÓN}

El artículo 29 de la convención sobre los derechos del niño hace referencia al derecho a la educación, la cual “deberá estar encaminada a: a) Desarrollar la personalidad, las aptitudes y la 
capacidad mental y física del niño hasta el máximo de sus posibilidades" (art. 21.a). Así esta educación integral, incluye la dimensión física y de salud, la cual, entre otras dimensiones, incluye la alimentación.

En los derechos a la infancia, además hay apartados específicos relacionados con la salud, que aquí enumeramos:

Art 24. "Los Estados Partes reconocen el derecho del niño al disfrute del más alto nivel posible de salud y a servicios para el tratamiento de las enfermedades y la rehabilitación de la salud".

Art 39.’Los Estados Partes adoptarán todas las medidas apropiadas para promover la recuperación física y psicológica y la reintegración social de todo niño víctima de: cualquier forma de abandono, abuso; tratos inhumanos o degradantes (...). Esa recuperación y reintegración se llevarán a cabo en un ambiente que fomente la salud, el respeto de sí mismo y la dignidad del niño".

Art. 17: "Los Estados Partes reconocen la importante función que desempeñan los medios de comunicación y velarán por que el niño tenga acceso a información (...), en especial la información y el material que tengan por finalidad promover su bienestar social, espiritual y moral y su salud física y mental".

En niño tiene "El derecho a salir de cualquier país" (art. 10), "El niño tendrá derecho a la liberta de expresión (art. 13)", Derecho de profesar la propia religión”, (art. 15), "libertad de asociación y de reuniones pacíficas" (art. 15), ahora bien, estos derechos tienen la restricción si fuera necesario para proteger la salud pública, por lo que el derecho a la salud está por encima de los anteriores derechos.

La adolescencia es una etapa en la que se presentan graves problemas relacionados con la alimentación, en concreto, la obesidad, bulimia y anorexia (trastorno, este último que pueden llevar a la muerte, y en muchos casos a deterioros neurológicos irreversibles). Así, pues, este es un tema de especial relevancia en la educación (Ayuso, 2019).

\section{ADOLESCENCIA Y SALUD.}

En general como afirma la OMS (2014a), se considera que los adolescentes son un grupo sano. A pesar de ello, muchos mueren de forma prematura debido a accidentes, suicidios, violencia, complicaciones relacionadas con el embarazo y enfermedades prevenibles o tratables. Más numerosos aún son los que tienen problemas de salud o discapacidades. Además, muchas enfermedades graves de la edad adulta tienen su origen en la adolescencia o en los hábitos incorrectos que se adquieren en ella. Por ejemplo, el consumo de tabaco, las infecciones de transmisión sexual, entre ellas el VIH, y los malos hábitos alimentarios y de ejercicio; causas todos ellos de enfermedad o muerte prematura en fases posteriores de la vida. 
La OMS señala los siguientes problemas como los más relevantes de la juventud: salud mental, consumo de sustancias, violencia, traumatismos involuntarios, nutrición, salud sexual y reproductiva y el VIH (OMS, 2014a).

En la misma línea, según la Resolución del Consejo y de los Representantes de los Gobiernos de los Estados de Europa (CSDH, 2008), sobre la salud y el bienestar de los jóvenes, aspectos como la nutrición, la actividad física, el abuso de alcohol y la salud sexual y mental son aspectos pendientes por su enorme repercusión en el estado de salud de los jóvenes europeos siendo esencial promover entre este grupo de población un modo de vida sano, adoptando medidas de prevención, teniendo en cuenta las cuestiones de género, y la relevancia de un clima relacional positivo (Bernárdez-Gómez, Álvarez-Muñoz y Belmonte, 2020).

\section{ALIMENTACIÓN Y ADOLESCENCIA}

La alimentación es un factor clave en el mantenimiento o la mejora del estado de bienestar de las personas. Junto con la actividad física es uno de los aspectos que intervienen de una forma determinante en la salud por su repercusión directa en la prevención de la obesidad y su asociación con las principales enfermedades crónicas de nuestro tiempo (cardiovasculares, diabetes mellitus tipo 2, hipertensión arterial y ciertos tipos de cáncer). De los diez factores de riesgo identificados por la OMS como claves para el desarrollo de las enfermedades crónicas, cinco están estrechamente relacionados con la alimentación y el ejercicio físico: obesidad, sedentarismo, hipertensión arterial, hipercolesterolemia y consumo insuficiente de frutas y verduras. Una alimentación poco saludable y no practicar actividad física con regularidad, son dos factores de riesgo importantes en la cronicidad de enfermedades y ambas son susceptibles de modificarse (Agencia Española de Seguridad Alimentaria, 2005; Moreno, 2015).

La prevalencia de sobrepeso y obesidad es una de las cuestiones que más preocupan en salud pública. Según los datos de la OMS, ambos han alcanzado carácter de epidemia mundial. El 65\% de la población mundial vive en países donde el sobrepeso y la obesidad se cobran más vidas de personas que la insuficiencia ponderal (estar por debajo del peso considerado como saludable) (OMS, 2014b).

Si bien el sobrepeso y la obesidad tiempo atrás eran considerados un problema propio de los países de ingresos altos, actualmente ambos trastornos están aumentando en los países de ingresos bajos y medios, en particular en los entornos urbanos. En los paises en desarrollo están viviendo cerca de 35 millones de niños con sobrepeso, mientras que en los países desarrollados esa cifra es de 8 millones. La causa fundamental del sobrepeso y la obesidad es un desequilibrio energético entre las calorías que se consumen y las calorías que se gastan. Pues bien, a nivel mundial se ha producido, por una parte, un aumento en la ingesta de alimentos hipercalóricos ricos en grasas, sal y azúcares, y pobres en vitaminas, 
minerales y otros micronutrientes. Por otra parte, se ha producido un descenso de la actividad física, como resultado de las formas cada vez más sedentarias de trabajo, de los nuevos modos de desplazamiento y de una creciente urbanización. A menudo los cambios en los hábitos de alimentación y actividad física son consecuencia de cambios ambientales y sociales asociados al desarrollo y la falta de políticas de apoyo en sectores como la salud, agricultura, transporte, planeamiento urbano, medio ambiente, educación, así como al procesamiento, distribución y comercialización de los alimentos (OMS, 2014b).

Ante la gran preocupación que la alimentación supone para la salud pública mundial, la OMS presentó la Estrategia Mundial sobre Régimen Alimentario, Actividad Física y Salud (OMS, 2004b) para intentar reducir los factores de riesgo de enfermedades no transmisibles relacionados con las dietas poco saludables y la inactividad física. En ella se insta a reforzar y establecer sistemas de vigilancia para evaluar la eficacia de las intervenciones realizadas y para orientar la inversión y gestión de los recursos disponibles reduciendo así los riesgos relacionados con los regímenes alimentarios poco sanos y la falta de actividad física. En 2006, el Marco para el seguimiento y evaluación de la aplicación de dicha Estrategia, propone una línea de actuación estableciendo indicadores que permiten evaluar la implantación de esta estrategia mundial (OMS, 2006).

La Oficina Regional para Europa de la Organización Mundial de la Salud, aprueba en 2007 el Plan de Acción Food and Nutrition Plan 2007-2009, en el que se plasma la necesidad de utilizar mecanismos de seguimiento y evaluación en las políticas y programas desarrollados comprobando así el logro de objetivos y estableciendo medidas basadas en la investigación y en la evidencia. En esta misma línea, se aprueba el Libro Blanco de la Unión Europea (2007), intentando contribuir a través de sistemas de seguimiento a la reducción de los problemas de salud relacionados con la mala alimentación, el sobrepeso y la obesidad.

En España, la dieta mediterránea y sus conocidos efectos positivos sobre la salud, instan al consumo de multitud y variedad de alimentos que forman parte de los hábitos alimentarios de nuestra población, lo cual predispone y favorece al menos el equilibrio nutricional. Se considera el modelo de alimentación más saludable y los beneficios sobre la salud que aporta la misma se han confirmado repetidamente (e.g., Estruch et al., 2013; Grao et al., 2013).

Numerosas investigaciones realizadas en los últimos años apuntan algunos hábitos de alimentación como los que mejor pueden ayudar al mantenimiento o mejora de la salud de las personas siempre en relación con la práctica de actividad física y como mejor modo de prevención de la obesidad y el sobrepeso (Moschonis et al., 2014; Papoutsou et al., 2014): ingesta adecuada de todos los tipos de alimentos de una forma variada y equilibrada; consumo ocasional de azúcares, alcohol y bebidas azucaradas; realizar entre tres y cinco comidas al día y realizar diariamente un desayuno completo (Pérez, 2019). 
En España, según la Encuesta Nacional de Salud (2013), el porcentaje de la población de entre 10 y 14 años afectados por obesidad y sobrepeso, ha aumentado un 7,1\% en los últimos 10 años. Mientras en 2003 el $34 \%$ de los adolescentes tenía sobrepeso u obesidad (29,3\% y 4,7\% respectivamente), este porcentaje asciende al $41,1 \%$ en 2012 (teniendo sobrepeso un 35,5\% y obesidad un 5,5\% de los adolescentes).

En 2005, el Ministerio de Sanidad y Consumo de España, preocupado por el fenómeno de la obesidad en nuestro país, elabora la Estrategia NAOS con la finalidad de mejorar los hábitos alimentarios e impulsar la práctica regular de la actividad física de todos los ciudadanos, poniendo especial atención en la prevención durante la etapa infantil. La puesta en marcha de esta estrategia ha requerido una intervención multisectorial, multidisciplinar y multifactorial interviniendo en los ámbitos familiar, escolar, empresarial y sanitario.

La citada estrategia nacional, ha seguido las directrices que la OMS establece en el Plan de Acción Europeo para la Alimentación y Política Nutricional 2007-2013, poniendo especial interés en el seguimiento y la evaluación como un componente ineludible de las políticas y planes de acción de prevención de la obesidad y de promoción de estilos de vida saludables. Así, en 2007, la OMS y la Asociación Española de Seguridad Alimentaria (AESAN), celebran en Madrid el Taller Internacional Estrategia Global de la OMS sobre Dieta, Actividad Física y Salud: un marco para la implantación de la evaluación y seguimiento, con el objetivo de revisar y compartir experiencias sobre evaluación en diferentes países, y discutir el desarrollo de planes de acción específicos para implantar el Marco para el seguimiento y evaluación de la aplicación de dicha Estrategia.

En la infancia y la adolescencia, la obesidad y el sobrepeso pueden causar hipertensión y complicaciones vasculares en etapas vitales posteriores, contribuyendo asimismo a la aparición de trastornos locomotores, complicaciones respiratorias, inmunológicas y gástricas así como diferentes alteraciones de la conducta como la merma de la autoestima y otros conflictos que pueden abocar al niño o al joven al fracaso escolar.

El estudio AVENA sobre alimentación y valoración del estado nutricional de los adolescentes españoles, muestra una alarmante prevalencia de sobrepeso y obesidad acompañada de bajos niveles de actividad y condición física y un estado metabólico de bajo grado, todos altamente implicados en el inicio y desarrollo de las enfermedades cardiovasculares que puede tener en un futuro este grupo de población (Wärnberg et al., 2006).

Haciendo referencia a la importancia de los hábitos de alimentación saludables, Contento y Michela (1998), demostraron que en el periodo de la adolescencia aumenta la necesidad fisiológica de los nutrientes esenciales (energía, proteínas, vitaminas y minerales) y se hace especialmente importante tener 
una dieta de alta calidad nutritiva. Un buen modelo de alimentación en la infancia y la adolescencia promueve un óptimo crecimiento y desarrollo, teniendo además un gran impacto en el futuro estado de salud adulto. Concretamente, una alimentación saludable en ambas etapas, previene problemas de salud inmediatos como la deficiencia de hierro, la anemia, la obesidad, desórdenes de alimentación y caries dental, al tiempo que todo lo anterior puede contribuir a prevenir problemas de salud a largo plazo, como la enfermedad coronaria, el cáncer, la hipertensión y la osteoporosis. Y, lo que no es menos importante, los hábitos de alimentación adquiridos en la adolescencia se convertirán en la adultez en rutinas que protejan la salud o que la pongan en riesgo (Moral et al, 2019).

Conviene señalar que hay cierta tendencia en esta etapa a una ingesta poco adecuada de picoteo y consumo de alimentos tipo "snacks" o de comida rápida de alto contenido calórico, ingestas desequilibradas o desordenadas en las que se come poco en las comidas porque se consumen alimentos entre horas. Además, es habitual que no se consuman alimentos necesarios como hidratos de carbono por miedo a engordar o que se prescinda de los desayunos. También se comienza en esta etapa a realizar dietas sin el control necesario de profesionales, lo cual ayuda a instaurar hábitos poco saludables y conduce en algunos casos a patologías como la anorexia y la bulimia (Ballesteros et al., 2011).

\section{EL DESAYUNO COMPLETO.}

Una de las formas más simples y eficaces de mejorar la salud y el bienestar es garantizar que los ciudadanos desayunen todos los días. Además de la influencia en el bienestar cotidiano, las investigaciones muestran que desayunar contribuye a mejorar la calidad de la ingesta diaria, al equilibrio metabólico y a la mejora del rendimiento cognitivo durante el día, lo cual implica beneficios para la salud a corto y largo plazo. Las investigaciones señalan que desayunar todos los días tiene una importante influencia positiva en el establecimiento de hábitos saludables, siendo una de las comidas más importantes de día y debiendo cubrir al menos el 25\% de las necesidades nutritivas diarias de los niños y adolescentes en edad escolar. La realización del desayuno está asociado con un menor riesgo de obesidad y una reducción en el índice de masa corporal en niños y adolescentes en Europa. Saltarse el desayuno, en cambio, se encuentra ligado a un estilo de vida poco saludable (Díaz, Ficapal y Aguilar, 2016; Ibarra, Mora, Ventura, y Hernández, 2019; Vereecken et al., 2009).

El estudio español AVENA muestra cómo los jóvenes que desayunan inadecuadamente o no desayunan, tienen unos hábitos menos saludables a lo largo del día (tanto en la comida como en la cena) (Moreno et al., 2005).

La OMS recomienda, para la realización de un desayuno completo con las mejores cualidades nutritivas, un lácteo (vaso de leche, yogur, queso...), una ración de cereal (pan, copos de cereales, 
galletas...), grasa de complemento (aceite de oliva, margarina o mantequilla, preferiblemente la primera) una fruta o un zumo de fruta, mermelada o miel y, sólo en ocasiones, otros alimentos como derivados cárnicos y dulces en cantidades moderadas.

A pesar de los beneficios del desayuno, las investigaciones muestran que saltárselo o realizarlo de manera insuficiente es un hecho cada vez más generalizado entre los niños y adolescentes (Vereecken et al., 2009). De Rufino et al. (2005), señalan que se trata de una práctica bastante frecuente entre los adolescentes. De hecho en población general, se ha comprobado que saltarse el desayuno es bastante más habitual en comparación con saltarse la comida o la cena general (Woodruff, Hanning, Lambraki, Storey y McCargas, 2008).

En España, resulta preocupante que el 9,45\% de los niños y jóvenes no desayunan nada y otro 30,3\% de este grupo de población desayunan solamente algo líquido como leche o zumo. Tan sólo un 26,4\% de la población infantil y juvenil española realiza un desayuno completo compuesto por leche, fruta o zumo de frutas y cereales (Encuesta Nacional de Salud, 2013).

Los resultados del estudio HBSC en España en su edición de 2010, aportaron información sobre el porcentaje de adolescentes de entre 11 y 18 años que desayunan los siete días de la semana siendo un $56,9 \%$ de los chicos y un 49,9\% de las chicas (Moreno et al., 2012). En Castilla y León un 13,9\% de adolescentes va al colegio sin desayunar siendo las chicas las que suben la media, un 17,4\% frente a un 9,6\% de chicos (Junta de Castilla y León, 2014).

\section{LA FRECUENCIA EN EL CONSUMO DE ALIMENTOS.}

En cuanto al consumo de los diferentes tipos de alimentos en la adolescencia, según La Agencia Española de Consumo, Seguridad Alimentaria y Nutrición (AECOSAN, 2007), resulta esencial para una alimentación saludable comer todo tipo de alimentos de una forma variada y equilibrada, tomar todos los días frutas verduras y hortalizas, cereales, patatas y legumbres, moderar el consumo de grasas especialmente de origen animal, moderar el consumo de productos ricos en azúcar y reducir la sal en las comidas (Aranceta, et al, 2016).

Los resultados de la Encuesta Nacional de Salud (2013) de España, muestran que el grupo de jóvenes de entre 15 y 24 años es el grupo poblacional que menos frutas y verduras incorpora en su dieta. Asimismo, numerosos estudios han demostrado el bajo porcentaje de consumo de estos dos grupos de alimentos en el periodo adolescente (e.g., Córdoba, Luengo y García, 2012; Martínez, Villarino, García, Calle y Marrodán, 2013), mientras que el consumo de dulces y refrescos crece de forma significativa en la última década (Legnani et al., 2012; Moreno-Gómez et al., 2012). 
En Castilla y León (España), alrededor del 10\% nunca toma alimentos como pescado, frutas frescas, verduras, legumbres y hortalizas. Por ejemplo, un 8,9\% de los encuestados no toma nunca frutas y cerca del $30 \%$ sólo la consumo una o dos veces por semana, siendo todavía peores los datos en verduras y hortalizas (un 9,7\% nunca las consume y un 43,2\% sólo una o dos veces por semana) (Junta de Castilla y León, 2014).

Además, en este estudio se refleja que hay un 71,5\% de los jóvenes que come mientras ve la televisión, lo que se relaciona con mayor ingesta y preferencia por platos más ricos en grasas y calorías vacías (Robinson et al., 2013). Si estos factores de riesgo se suman a la baja frecuencia de ejercicio físico y el tiempo dedicado a las conductas sedentarias (tal y como se tratará en el siguiente apartado), se muestra un patrón a tener en cuenta en la prevención del sobrepeso y la obesidad en la edad adolescente.

Los datos del estudio HBSC en España (Moreno et al., 2010), muestran que en la realización de un desayuno completo, el porcentaje de adolescentes que desayuna los siete días de la semana es mayor en chicos $(56,9 \%)$ que en chicas $(49,9 \%)$. Además la tendencia descendente de esta práctica según la edad es más acusada en las chicas. En cuanto al consumo de los distintos grupos de alimentos, los datos apuntan que las chicas superan a los chicos en consumo óptimo de frutas y realizan también un mayor consumo diario de verduras. En cuanto al consumo diario de refrescos o bebidas azucaradas el porcentaje es más frecuente en los chicos, un $24,4 \%$ frente al $20,2 \%$ de las chicas. Los dulces, en cambio, son consumidos a diario de forma más frecuente por las chicas $(16,6 \%)$ frente al $13,7 \%$ de los chicos.

Un estudio realizado por nuestro equipo (Guevara, 2014) mostró que en una muestra de 1200 adolescentes españoles, el $71 \%$ desayunaba de forma completa todos los días, pero un $12 \%$ de los adolescentes encuestados no desayunaba nunca. El hábito no saludable de alimentación más frecuente es el bajo consumo de verduras y frutas, y el excesivo en carne. Además, el consumo de azúcares y bebidas azucaradas es alto en este grupo de población. Tan sólo un 12\% de los adolescentes cumple las recomendaciones realizadas por los organismos internaciones para seguir una alimentación equilibrada y completa.

\section{CONCLUSIÓN}

Los datos señalados en esta revisión ponen de manifiesto la necesidad de diseñar programas de intervención que de forma específica promuevan un mayor consumo de frutas y verduras, la realización de un desayuno completo todos los días de la semana y además orienten sobre la frecuencia recomendada en el consumo de alimentos" (Guevara, p. 208). De entre los factores que determinan el estado de salud, cobra especial relevancia la alimentación y sobre todo en la etapa adolescente ya que durante la misma se 
adquieren muchos de los hábitos que nos acompañan a lo largo de la vida. La salud es un derecho en la infancia y promoverla favorece la educación integral de las personas. 


\section{REFERENCIAS}

Agencia Española de Seguridad Alimentaria (2005). Estrategia NAOS. Invertir la tendencia de la obesidad. Estrategia para la nutrición, actividad física y prevención de la obesidad. Madrid: Ministerio de Sanidad y Consumo

AECOSAN- Agencia Española de Seguridad Alimentaria y Nutrición (2007). La alimentación en Educación Secundaria Obligatoria. Madrid: Ministerio de Sanidad y Consumo.

Aranceta, J, Arija, V., Maíz, E., Martínez, E., Ortega, R.M., Pérez-Rodrigo, C, Quiles, J, Rodríguez, A., Román, B., Salvador i Castell, G., Tur JA, Varela, G. y Serra, L. (2016). Guías alimentarias para la población española (SENC); la nueva pirámide de la alimentación saludable. Nutrición Hospitalaria, 33 (8), 1-48.

Ayuso, R., Molina, M.C. y Medina, J.L (2019). La promoción de salud en la escuela como estrategia para el abordaje de la cronicidad en España. Horizonte Sanitario,18 (1), 37-47.

Ballesteros, J. M., Pérez, N., Quiles, J., Echeverría, P., Castell, C., Muñoz, J., Mosquera, C., Belmonte, S. Rivas, F., Gómez, F., Calvo, A., Martínez, R., y Lizalde, E. (2011). Evaluación y seguimiento de la Estrategia Naos: conjunto mínimo de indicadores. Madrid: Ministerio de Sanidad, Política Social e Igualdad.

Bernárdez-Gómez, A., Álvarez-Muñoz, J.S. y Belmonte, M.L. (2020). La honestidad, la amistad y la autoestima: valores que promueven el clima relacional positivo. South Florida Journal of Development, 2(1),119-130.

Contento, I. R., y Michela, J. W. (1998). Nutrition and food choice behavior among Children and adolescentes. En Gorreczny y Hensen (eds.) Handbook of pediatric and adolescent health psychology (pp. 249-273). Boston: Allyn \& Bacon.

Córdoba, L. G., Luengo, L. M, y García, V. (2012). Ingesta dietética de los estudiantes de secundaria de la ciudad de Badajoz. Endocrinología y Nutrición, 59(7), 407-415.

De Rufino, P., Redondo, C., Amigo, T., González-Lamuño, D., García, M. y Grupo AVENA. (2005). Desayuno y almuerzo de los adolescentes escolarizados de Santander. Nutrición Hospitalaria, 20, $217-$ 222.

Díaz, T, Ficapal, P. y Aguilar, A. (2016). Hábitos de desayuno en estudiantes de primaria y secundaria. Posibilidades para la educación nutricional en la escuela. Nutrición Hospitalaria, 33 (4), 909-914.

Ibarra, J., Mora, L., Ventura, C. y Hernández, C. (2019). Hábitos de vida saludable de actividad física, alimentación, sueño y consumo de tabaco y alcohol, en estudiantes adolescentes chilenos. Revista Técnico-Científica del Deporte Escolar, Educación Física y Psicomotricidad, 5(1), 70-84.

Instituto Nacional de Estadística (2013). Encuesta Nacional de Salud de España.

Estruch, R., Ros, E., Salas-Salvadó, J., Covas, M.I., Corella, D., Arós, F., Gómez-Gracia, E., RuizGutiérrez, V., Fiol, M., Lapetra, J., Lamuela-Raventos, R. M., Serrano-Majem, M. D., Pitó, X., Basora, J., Muñoz, M. A., Martínez, J. A. y Martínez-González, M. A. (2013). Primary Prevention of Cardiovascular Disease with a Mediterranean Diet. The New England Journal of Medicine, 368, 14. 
Guevara, R. (2014). Estilos de vida relacionados con la salud de los adolescentes salmantinos. Tesis doctoral. Universidad Pontificia de Salamanca (España).

Grao, A., Nuviala, A., Fernández, A., Porcel, A. M., Moral, J. E. y Martínez, E. J. (2013) Adherencia a la dieta mediterránea en adolescentes rurales y urbanos del sur de España, satisfacción con la vida, antropometría y actividades físicas y sedentarias. Nutrición Hospitalaria, 28(3), 1129-1135.

Grosso, G., Marventano, S., Buscemi, S., Scuderi, A., Matalone, M., Platania, M., Giorgianni, G., Rametta, S., Nolfo, F., Galvano, F. y Mistretta, A. (2013). Factors Associated with Adherence to the Mediterranean Diet among Adolescents Living in Sicily, Southern Italy. Nutrients, 5(12), 4908-4923.

Junta de Castilla y León (2014). Sistema de Vigilancia de los hábitos y estilos de vida en la población juvenil de Castilla y León. Notas de prensa: Diario de Burgos.

Legnani, E., Legnani, R. F., Dellagrana, R. A., Siva, M. P., Barbosa, V. C. y Campos, W. (2012). Healthrelated risk behaviors and overweight in students from Toledo, Paraná, Brazil. Motricidade, 8(3), 59-70.

Martínez, J. R., Villarino, A., García, R. M., Calle, M. E. y Marrodan, M. D. (2013). Obesidad infantil en España: hasta qué punto es un problema de salud pública o sobre a fiabilidad de las encuestas. Nutrición Clínica y Dietética Hospitalaria, 33(2), 80-88.

Moral, J.E., Agraso, A.D., Pérez, J.J., Rosa, A., Tárraga, M.L., García, E. y Tárraga, P.J. (2019). Práctica de actividad física según adherencia a la dieta mediterránea, consumo de alcohol y motivación en adolescentes Nutrición Hospitalaria, 36(2), 420-427.

Moreno, L. A., Kersting, M., de Henauw, S., González-Gross, M., Sichert-Hellert, W., Matthys, C., Mesana, M. I. y Ross, N. (2005). How to measure dietary intake and food hábits in adolescence: The European perspective. International Journal of Obesity, 29, 66-77.

Moreno, C., Ramos, P., Rivera, F., Jiménez-Iglesias, A., García-Moya, I., Sánchez-Queija, I., López, A. y Granado, M. C. (2012). Las conductas relacionadas con la salud y el desarrollo de los adolescentes españoles. Resultados del Estudio HBSC-2010 con chicos y chicas españoles de 11 a 18 años. Madrid: Ministerio de Sanidad.

Moreno, L.A. (2015). La alimentación del adolescente. Mediterráneo Económico (27), 75-86.

Moschonis, G., Kalliora, A. C., Costarelli, V., Papandreou, C., Koutoukidis, D., Lionis, C., Chrousos, G. P., Manios, Y. y Healthy Growth Study Group (2014). Identification of lifestyle patterns associated with obesity and fat mass in children: the Healthy Growth Study. Public Health Nutrition, 17(3), 614-624.

Organización Mundial de la Salud (2006). Marco para el seguimiento y evaluación de la aplicación de la Estrategia Mundial sobre Régimen Alimentario, Actividad Física y Salud. Ginebra: OMS.

Organización Mundial de la Salud (2014a). Salud del adolescente.

Organización Mundial de la Salud (2014b). Obesidad y sobrepeso. Nota descriptiva, 311. Mayo.

Papoutsou, S., Briassoulis, G., Hadjigeorgiou, C., Savva, S. C., Solea, T., Hebestreit, A., Pala,V., Sieri, S., Kourides, Y., Kafatos, A. y Tornaritis, M. (2014). The combination of daily breakfast consumption 
and optimal breakfast choices in childhood is an important public health message. International Journal of Food Sciences and Nutrition, 65(3), 273- 279.

Pérez Hernández, I. (2019). Nutrición, alimentación y estilos vida en los niños y adolescentes. Cuadernos de pedagogía, 497, 80-83.

Commission on Social Determinants of Health [CSDH] (2008). Closing the gap in a generation: health equity through action on the social determinants of health. Final Report of the Commission on Social Determinants of Health. Geneva: WHO.

Robinson, E., Aveyard, P., Daley, A., Jolly, K., Lewis, A., Lycett, D. y Higgs, S. (2013). Eating attentively: a systematic review and meta-analysis of the effect of food intake memory and awareness on eating. The American Journal of Clinical Nutrition, 97 (4), 728-742.

UNICEF Comité Español (2006). Convención sobre los derechos del niño. Madrid: UNICEF.

Vereecken, C. Dupuy, M., Rasmussen, M., Kelly, C., Nansel, T.R., Al Sabbah, H., Baldassari, D., Delgrande, M., Maes, L., Niclasen, B., Ahluwalia, N. y the HBSC Eating y Dieting Focus Group (2009). Breakfast consumption and its socio-demographic and lifestyle correlates in schoolchildren in 41 countries participating in the HBSC study. Internacional Journal of Public Health, 54, 180-190.

Wärnberg, J., Ruiz, J. R., Ortega, F. B., Romeo, J., González-Gross, M., Moreno, L.A., García-Fuentes, M., Gómez, S., Nova, E., Díaz, L.E., Marcos, A. y Grupo AVENA (2006). Estudio AVENA (Alimentación y valoración del estado nutricional en adolescentes). Resultados obtenidos 2003-2006. Pediatría Integral, 1, 50-55.

Woodruff, S. J., Hanning, R. M., Lambraki, I., Storey, K. E. y McCargas, L. (2008). Healthy Eating Index$\mathrm{C}$ is compromised among adolescent with body weight concerns, weight loss dieting, and meal skipping. Body Image, 5, 404-408. 